\title{
A fully relativistic lattice Boltzmann algorithm
}

\author{
P. Romatschke, 田 M. Mendoza, ${ }^{2, \text { U }}$ and S. Succi ${ }^{3}$, 团 \\ ${ }^{1}$ Frankfurt Institute for Advanced Studies, D-60438 Frankfurt (Germany) \\ ${ }^{2}$ ETH Zürich, Computational Physics for Engineering Materials, \\ Institute for Building Materials, Schafmattstrasse 6, HIF, CH-8093 Zürich (Switzerland) \\ ${ }^{3}$ Istituto per le Applicazioni del Calcolo C.N.R., Via dei Taurini, 19 00185, Rome (Italy), \\ and Freiburg Institute for Advanced Studies, Albertstrasse, 19, D-79104, Freiburg, Germany
}

(Dated: April 1, 2022)

\begin{abstract}
Starting from the Maxwell-Jüttner equilibrium distribution, we develop a relativistic lattice Boltzmann (LB) algorithm capable of handling ultrarelativistic systems with flat, but expanding, spacetimes. The algorithm is validated through simulations of quark-gluon plasma, yielding excellent agreement with hydrodynamic simulations. The present scheme opens the possibility of transferring the recognized computational advantages of lattice kinetic theory to the context of both weakly and ultra-relativistic systems.
\end{abstract}

PACS numbers: 47.75.+f, 47.11.-j

Keywords: Relativistic fluid dynamics, Quark-gluon plasmas, Lattice Boltzmann

\section{MOTIVATION}

The great success of the Relativistic Heavy-Ion Collider (RHIC) experimental program [1-4] has provided the motivation to come up with realistic and quantitative simulations of heavy-ion collisions.

Since the bulk of particles produced in relativistic heavy-ion collisions is described by fluid dynamics [5], the center-piece of any complete simulation attempt will involve a viscous fluid dynamics algorithm. The majority of presently available fluid dynamics codes is able to handle smooth initial conditions in $2+1$ dimensions in the presence of shear viscosity [6-10]. However, it has by now been understood that the presence of event-by-event fluctuations in the initial state can lead to significantly different quantitative results with respect to smooth initial conditions [11], and may in some cases even explain qualitatively new phenomena. To be more specific, the presence of event-by-event fluctuations is the source of the non-vanishing elliptic flow found in RHIC experiments at central collisions, the source of hydrodynamic flow-fluctuations, and may (through the presence of socalled triangular flow $v_{3}$ ) naturally explain the presence of the 'ridge phenomenon' found in experiments [12 15]. Thus, it is probably fair to say that without including the effect of event-by-event fluctuations, a description of the medium created in heavy-ion collisions cannot be regarded as realistic. This provides the motivation to develop a fully relativistic and computationally efficient viscous fluid dynamics algorithm that can handle initial state fluctuations. Also, such an algorithm can be used to validate the only available $3+1$ dimensional relativistic viscous hydrodynamics code by the McGill group [16].

\footnotetext{
* romatschke@fias.uni-frankfurt.de

$\dagger$ mmendoza@ethz.ch

$\ddagger$ succi@iac.cnr.it
}

Further motivation is provided by other systems where relativistic viscous fluid flows are of interest, such as astrophysical systems and condensed matter systems such as graphene [17]. One particular question that arises in all this different systems is when relativistic fluid flow becomes turbulent, which involves a determination of the critical Reynolds number and the turbulent spectrum [18, 19].

\section{LATTICE KINETIC APPROACH TO HYDRODYNAMICS}

Fluid turbulence, both classical and relativistic, sets one of the most compelling challenges in modern computational physics. This motivates a relentless search for new and ever more efficient methods for solving the hydrodynamic equations of motion in the high-Reynolds turbulent regimes. In the last two decades, a new computational paradigm has emerged, which is based on the idea of solving hydrodynamic problems from the standpoint of Boltzmann kinetic theory. Apparently, this is rather counterintuitive, because the Boltzmann equation lives in a double-dimensional (phase) space, consisting of three dimensions in ordinary space, plus three additional dimensions in momentum (velocity space). In addition, the Boltzmann equation contains the details of microscopic interactions through a very complicated integral collision operator in velocity space, which is computationally very demanding. As a result, the Boltzmann equation has never been considered a practical tool for computational fluid dynamics, apart from the special case of rarefied gas dynamics, for which ordinary fluid dynamics is known to be inadequate. In the last two decades, however, minimal realizations of the Boltzmann equation have been developed, which relinquish the aforementioned problems, and gave rise to a computational method of remarkable elegance and outstanding computational efficiency. Since these minimal forms of 
Boltzmann kinetic equations are formulated in a discrete velocity and space-time lattice, they have come to be known as Lattice Boltzmann Equation(s) (LBE) [28 30]. To date, LB methods have met with amazing success across virtually all sectors of non-relativistic fluid dynamics, from flows to porous media, to turbulent flows in complex geometries, multiphase, colloidal, hemodynamic flows, and magnetohydrodynamics 31 38]. However, relativistic formulations have come into existence only very recently [39, 40]. Indeed, the lattice formulation of the relativistic Boltzmann equation presents a series of theoretical and methodological challenges which have no counterpart in the non-relativistic realm. To date, some of these challenges have been bypassed by formulating a relativistic LBE (RLBE) top-down, i.e. by recovering the equations of relativistic hydrodynamics through a moment-matching procedure in the lattice. This gives rise to a very efficient computational scheme for mildly relativistic systems, but does not guarantee the so-called "realizability" of RLBE, i.e. the fact that RLBE should be derived by an underlying microscopic model, or, at least, by a continuum version of a relativistic kinetic equation. Even leaving aside computational considerations, this is an important task in the process of placing the RLBE onto a solid conceptual framework. This is precisely the task accomplished in the present paper.

\section{RELATIVISTIC KINETIC THEORY}

Before discussing the details of LB methods, let us briefly review the theoretical background of kinetic theory in a relativistic context. The starting point is the Boltzmann equation for the single particle distribution $f=f\left(x^{\mu}, p^{\alpha}\right)$, with the relativistic analogue of the Bhatnagar-Gross-Krook collision term [41]:

$$
\left[p^{\mu} \nabla_{\mu}-\Gamma_{\mu \nu}^{\lambda} p^{\mu} p^{\nu} \partial_{\lambda}^{(p)}\right] f=-\frac{p^{\mu} u_{\mu}}{\tau_{R}}\left(f-f_{J}^{\mathrm{eq}}\right),
$$

Here $x^{\mu}$ and $p^{\alpha}$ are the position and momentum 4vectors, respectively, $\tau_{R}$ is the single relaxation time, and $f_{J}^{\text {eq }}$ denotes the equilibrium distribution function. Here and in the following, we work in units where the speed of light $c$, the Boltzmann constant $k_{B}$ and Planck's constant $\hbar$ have been set to unity, $c=k_{B}=\hbar=1$. In the ultrarelativistic case, this may be taken in the form of the Jüttner distribution function

$$
f_{J}^{\mathrm{eq}}=Z^{-1} e^{-p_{\mu} u^{\mu} / T}
$$

where $Z^{-1}$ parametrizes the number of degrees of freedom, $u^{\mu}$ is the macroscopic (fluid) 4 -velocity, $T$ is the local temperature, $\nabla_{\mu}$ denotes the (geometric) covariant derivative, and $\Gamma_{\mu \nu}^{\lambda}$ are the Christoffel symbols that are given by derivatives of the underlying metric tensor $g_{\mu \nu}$.

The connection to fluid dynamics is realized by intro- ducing the energy-momentum (energy-stress) tensor $T^{\mu \nu}$,

$$
\begin{aligned}
T^{\alpha \beta}(t, x) & \equiv \int d \chi p^{\alpha} p^{\beta} f(t, x, p) \\
& \equiv \int \frac{d^{4} p}{(2 \pi)^{3}} \delta\left(p^{\mu} p_{\mu}-m^{2}\right) 2 H\left(p^{0}\right) p^{\alpha} p^{\beta} f(t, x, p),
\end{aligned}
$$

where $m$ is the particle mass and $H$ the Heavyside step function. Note that we have introduced the notation $f(t, x, p) \equiv f\left(x^{\mu}, p^{\alpha}\right)$. The equations of motion obeyed by the tensor $T^{\mu \nu}$ emerge, after a little algebra, upon integrating Eq. (11) with respect to four-momentum degrees of freedom, $\int d \chi p^{\nu}$,

$$
\nabla_{\mu} T^{\mu \nu}=-u_{\mu} \int d \chi p^{\mu} p^{\nu} \frac{\left(f-f_{\mathrm{eq}}\right)}{\tau_{R}}=-\frac{u_{\mu}}{\tau_{R}}\left(T^{\mu \nu}-T_{\mathrm{eq}}^{\mu \nu}\right) .
$$

The equilibrium energy-momentum tensor $T_{\text {eq }}^{\mu \nu}$ is readily computed using the Jüttner distribution, and reads as follows:

$$
T_{\mathrm{eq}}^{\mu \nu}=(\epsilon+P) u^{\mu} u^{\nu}-P g^{\mu \nu},
$$

where the energy density $\epsilon$ and pressure $P$ are functions of the temperature and the number of degrees of freedom $Z$ (here arbitrarily set to one). The full energy momentum tensor may then be written as $T^{\mu \nu}=T_{\mathrm{eq}}^{\mu \nu}+\Pi^{\mu \nu}$, where the second term collects non-equilibrium contributions.

Still needed is the choice of the rest-frame of the heat bath with respect to which the fluid velocity $u^{\mu}$ is defined. In the ultrarelativistic limit, the canonical choice is the so-called Landau-Lifshitz condition, whereby $u_{\mu} T^{\mu \nu} \equiv$ $\epsilon u^{\nu}$, so that Eq. (4) reads simply as:

$$
\nabla_{\mu} T^{\mu \nu}=0,
$$

expressing the (covariant) conservation of energy and momentum. Sufficiently close to equilibrium, where gradients are small, the form of $\Pi^{\mu \nu}$ can be calculated by integrating Eq. (1) with respect to $\int d \chi p^{\mu} p^{\alpha}$ (c.f. [20, 21]). In the ultrarelativistic case, when particle masses can be neglected $(m=0)$ one finds:

$$
\Pi^{\alpha \beta}=\tau_{R} \frac{\epsilon+P}{6} \nabla^{<\alpha} u^{\beta>},
$$

where

$$
A^{<\alpha} B^{\beta>} \equiv\left(\Delta^{\alpha \mu} \Delta^{\beta \nu}+\Delta^{\alpha \nu} \Delta^{\beta \mu}-\frac{2}{3} \Delta^{\alpha \beta} \Delta^{\mu \nu}\right) A_{\mu} B_{\nu},
$$

and $\Delta^{\mu \nu}=g^{\mu \nu}-u^{\mu} u^{\nu}$. Performing a non-relativistic limit of $\nabla_{\mu} T^{\mu \nu}$ one recovers the Navier-Stokes equations, with the following dynamic viscosity coefficient

$$
\eta=\tau_{R} \frac{\epsilon+P}{6} .
$$

Therefore, Eq. (11) reproduces the equations of fluid dynamics in the continuum, on condition that $\tau_{R}=6 \frac{\eta}{s} T^{-1}$, 
where $s$ denotes the entropy density and gradients must be small enough that a fluid dynamics description makes sense at all. A few remarks are in order: by construction, kinetic theory achieves a remarkable disentangling between non-linearity and non-locality, which proves beneficial for both theoretical and computational purposes. Indeed, in the hydrodynamic formulation, any generic quantity, including the flow velocity, is transported along space-time changing trajectories, defined by the flow velocity itself, thereby giving rise to terms which are non-local and non-linear at a time. In a turbulent flow, such trajectories become typically fairly complicated, thus opening potential exposures to numerical inaccuracies and instabilities. In kinetic theory, on the contrary, information is always transported along constant characteristics, $d x=v d t$, since the velocity $v$ (vector notation relaxed for simplicity) does not depend on space-time coordinates. Thus, no matter how wild the space-time dependence of the fluid flow, the streaming operator is linear in the Boltzmann distribution function, and the information always travels along straight lines. The price to pay for this major advantage is the need of three extra-dimensions in velocity space. However, velocity space lends itself to very economic discretizations, typically $O\left(3^{d}\right)$ discrete velocities in $d$ spatial dimensions space, which make the tradeoff between non-linearity and over-dimensionality an excellent bargain, and is one of the key assets of the LB formulation.

One may then wonder where the non-linearity has disappeared in the kinetic formulation. It turns out that it is concealed in the local equilibrium distribution, which is a non-linear function of the local hydrodynamic variables, see expression (2). Furthermore, note that since collisions are zero-ranged in the Boltzmann picture, the corresponding collision term is completely local, as anticipated. This lies at the root of the excellent amenability of LB to parallel computing, another major practical asset of the technique altogether 32].

Having clarified the main philosophy of the kinetic pathway to fluid dynamics, one must come down to specific details. The main question is: how sparse can the sampling in momentum space be made?

The target criterion is that $\nabla_{\mu} T^{\mu \nu}=0$ must emerge as a continuum limit of the lattice analogue of Eq. (1). Like in the non-relativistic framework, this sets a specific demand on the symmetry of the lattice tensors, as detailed in a sequel to this work. In particular, second order tensorial identities of the form

$$
u_{\mu} \int d \chi p^{\nu} p^{\mu} f(t, x, p)=\epsilon u^{\nu} .
$$

have to be reproduced exactly in the lattice formulation.

\section{DETOUR: THE NON-RELATIVISTIC LATTICE BOLTZMANN FORMULATION}

At this point, it is instructive to review the setup of the LB scheme in the non-relativistic context. There, the equilibrium distribution function for an ideal gas is the Maxwell distribution,

$$
f_{M}^{\mathrm{eq}}(t, x, v)=\frac{\rho(t, x)}{\sqrt{2 \pi c_{s}^{2}}} e^{-\frac{(\mathrm{v}-\mathrm{u})^{2}}{2 c_{s}^{2}}},
$$

where $c_{s}=\sqrt{T / m}$ is the sound speed, the velocities $\mathbf{v}$ (alternatively denoted as $v^{i}$ with $i$ running on spatial coordinates) take the role of the momenta $p$, and $\mathbf{u}$ is the macroscopic velocity. By introducing a scaled temperature $\theta=\frac{T}{T_{0}}, T_{0}$ being a reference temperature, and rescaling the velocities with the speed of sound, the velocity moments (the conditions to recover fluid dynamics corresponding to Eq. (5)), take the form [22]

$$
\begin{gathered}
\int d^{3} v f(t, x, v)=\rho, \quad \int d^{3} v \mathbf{v} f(t, x, v)=\rho \mathbf{u}, \\
\int d^{3} v \mathbf{v}^{2} f(t, x, v)=2 \rho \epsilon_{\mathrm{int}}+\rho \mathbf{u}^{2},
\end{gathered}
$$

where $\rho$ is the mass density and $\epsilon_{\text {int }}$ the internal energy density. Note that the local Maxwellian corresponds to the generating functional of the Hermite polynomials $H_{n}(v)$,

$$
e^{-(v-u)^{2} / 2}=e^{-v^{2} / 2} \sum_{n} H_{n}(v) \frac{u^{n}}{n !},
$$

in one-dimension. This is readily generalized to three dimensions, thanks to the factorizability of the prefactor $e^{-\mathbf{v}^{2} / 2}$ into the respective components. The actual Maxwellian distribution can then be approximated as

$$
e^{-(v-u)^{2} / 2 \theta} \sim e^{-v^{2} / 2 \theta} \sum_{n=0}^{N} \frac{a_{n}\left(u / \theta^{1 / 2}\right)}{n !} H_{n}\left(v / \theta^{1 / 2}\right),
$$

which is valid in the sense of mean convergence, if

$$
\int d \mathbf{v} e^{v^{2} / 2} e^{-|\mathbf{v}-\mathbf{u}|^{2} / 2 \theta}
$$

exists, or equivalently $\theta<2$ (c.f. [22, 23]). The same representation can be applied to the full distribution $f(t, x, v)$, which reads (after rescaling $v \rightarrow v \theta^{1 / 2}$, $\left.u \rightarrow u \theta^{1 / 2}\right)$ :

$$
f(t, x, v)=e^{-v^{2} / 2} \lim _{N \rightarrow \infty} \sum_{n=0}^{N} \frac{a_{n}^{i_{1} \ldots i_{n}}(t, x)}{n !} \mathcal{H}_{n}^{i_{1} \ldots i_{n}}(\mathbf{v}),
$$

where $\mathcal{H}_{n}^{i_{1} \ldots i_{n}}$ are tensor Hermite polynomials (c.f. [23]). In practice, $f$ will be approximated by truncating the above sum at finite (small) $N$.

Integrals of the form $\int e^{-v^{2} / 2} P(\mathbf{v}) d \mathbf{v}$, where $P$ is a polynomial of degree $2 N$ or less, can then be calculated exactly as a sum over the roots of $H_{N}(v)$ ("GaussHermite quadrature"). Therefore, the roots $v=v_{m}$, 

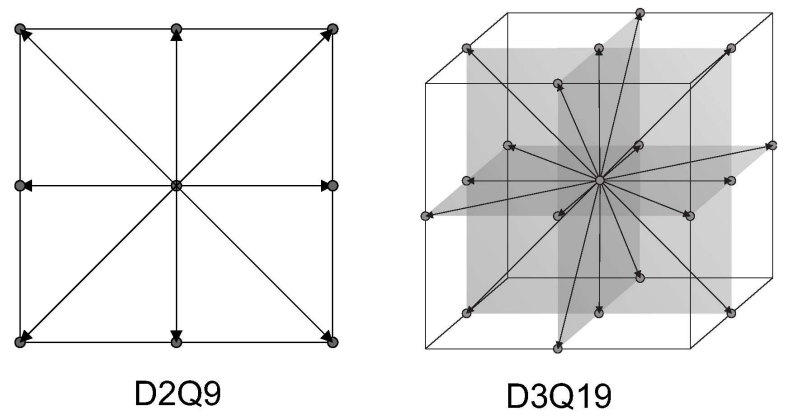

FIG. 1. Typical lattice configurations D2Q9 (9 velocities in 2 dimensions) and D3Q19 (19 velocities in 3 dimensions) for the lattice Boltzmann model. The arrows denote the discrete unit vectors set.

$m=1, \ldots N$ represent the ideal choice for the discretization/sampling of velocity space. To guarantee that the Boltzmann equation reproduces the non-relativistic fluid dynamics equations, the velocity moments must be represented exactly, which implies the necessary condition $N \geq 2$. For many applications, specifically those not dealing with strong thermal and compressible phenomena, this is also sufficient to a second order numerical accuracy. The end result is a discrete (lattice) Boltzmann equation of the form

$$
f_{i}\left(t+\Delta t, \mathbf{x}+\mathbf{c}_{i} \Delta t\right)-f_{i}(t, \mathbf{x})=-\Omega\left(f_{i}-f_{i}^{e q}\right),
$$

where $\Omega=\frac{\Delta t}{\tau_{R}}$ with $\tau_{R}$ the single relaxation time. The discrete velocities $\mathbf{c}_{i}$ run over a lattice with sufficient symmetry to guarantee mass, momentum and momentumflux conservation (6) , so as to recover the exact form of the Navier-Stokes equations. Typical lattices fulfilling the above constraints are the D2Q9 (nine velocities in two dimensions) and D3Q19 (nineteen speeds in three dimensions), see Fig. 1 .

Note that, owing to the Cartesian formulation, these lattice configurations are space-filling. This is crucial to ensure the so called light-cone condition $d \mathbf{x}_{i}=\mathbf{c}_{i} d t$, i.e. the discrete populations hop from site to site in fully synchronous mode (grid-bound dynamics), a feature which turns out to be critical to the computational efficiency of the LB scheme. Upon performing a standard Chapman-Enskog asymptotic expansion, the lattice Boltzmann scheme $([28-30])$ is shown to recover the hydrodynamic equations of a quasi-incompressible fluid with kinematic viscosity (in lattice units $\Delta t=\Delta x=1$ ) $\nu=c_{s}^{2}\left(\tau_{R}-1 / 2\right), c_{s}$ being the lattice sound speed, typically $1 / \sqrt{3}$. To be noted, the factor $-1 / 2$ at the right hand side, which stems from a second order Taylor expansion of the discrete streaming LBE operator. This term, known as 'propagation viscosity', contributes a negative viscosity and permits to achieve very small viscosities $\nu \ll 1$ with unit time-steps, by simply choosing $\tau_{R}=1 / 2+\nu / c_{s}^{2}$. This property is crucial to access low-viscous, turbulent regimes while still preserving an efficient time-marching procedure.

\section{A FULLY RELATIVISTIC LB ALGORITHM}

The lattice formulation of relativistic kinetic theory poses a few genuinely new challenges, primarily the fact that the energy $E$ is no longer a simple quadratic function of the velocity (momentum) $E=\sqrt{m^{2}+\mathbf{p}^{2}}$. This basic feature reflects in the non-separability of the Jüttner distribution along the three components of the momentum $\mathbf{p}$, and forbids a simple transcription of the Hermite procedure described above for the case of non-relativistic fluids.

This is the reason why the only existing relativistic LBE version available to date is based on a top-down procedure, i.e. design lattice equilibria with free Lagrangian parameters, which are then adjusted in such a way as to match the five basic conservation laws, number density and energy-momentum 4-vector. Full details can be found in the original references [39, 40]. The scheme was validated for two different relativistic applications, 1d quark-gluon plasmas, 3d supernova explosions, and graphene [42], showing excellent performance on all of them. However, inherent to the moment-matching procedure, is the question of realizability, i.e. the existence of an underlying microscopic model or at least an equivalent analogue in continuum kinetic theory. Moreover, the top-down procedure only works in the case of Cartesian coordinates. In view of general relativistic applications, involving generic coordinate systems, it is highly desirable to explore the viability of the relativistic LB procedure beyond the Cartesian realm (incidentally, this would prove useful also for non-relativistic applications in general coordinates).

For the ultra-relativistic case, where particle masses can be neglected, the equilibrium distribution reads as

$$
e^{-p \cdot u / T}=e^{-|\mathbf{p}| u^{0} / T+\mathbf{p} \cdot \mathbf{u} / T},
$$

which does not allow an ansatz such as Eq. (7) because the pre-factor corresponds to an unfactorizable square root dependence. This alone prevents a straightforward use of Cartesian coordinates.

Rather, the equilibrium distribution function suggests the following expansion:

$$
e^{-p \cdot u / T}=e^{-|\mathbf{p}| u^{0} /\left(T_{0} \theta\right)} \sum_{n}\left(\frac{\mathbf{p}}{|\mathbf{p}|}\right)^{n}\left(\frac{|\mathbf{p}| u^{0}}{T_{0} \theta}\right)^{n} \frac{(\mathbf{u})^{n}}{\left(u^{0}\right)^{n} n !}
$$

which involves unit vectors $\mathbf{v}=\frac{\mathbf{p}}{|\mathbf{p}|}$ rather than momenta $\mathbf{p}$ and powers of $\frac{|\mathbf{p}| u^{0}}{T_{0} \theta}$, that go together with the exponent and where again the scaled temperature $\theta=\frac{T}{T_{0}}$ was introduced.

This leads to the following ansatz for the relativistic distribution function $f$ :

$$
f(t, x, p)=e^{-p^{0} / T_{0}} \sum_{n} P_{i_{1} \ldots i_{n}}^{(n)}(\mathbf{v}) a_{i_{1} \ldots i_{n}}^{(n)}\left(t, x, p^{0} / T_{0}\right),
$$


where $p^{0}=|\mathbf{p}|$ and vector polynomials $P_{i_{1} \ldots i_{n}}^{(n)}(\mathbf{v})$ which are orthogonal with respect to the angular integral $\int \frac{d \Omega}{4 \pi}$. Their properties are listed in appendix $\mathrm{A}$.

The ansatz (12) should constitute a valid approximation to $f(t, x, p)$ in the sense of mean convergence, i.e. provided that the integral

$$
\int_{0}^{\infty} d p^{0} e^{p^{0} / T_{0}} f^{2}(t, x, p)
$$

exists, which together with Eq.(10) implies $\theta<$ $2\left(u^{0}-|\mathbf{u}|\right)$. For highly relativistic fluid flows such as those with Lorentz factor of $\gamma \sim 10, \theta=0.1$ would ensure convergence. However, in practical applications, such as the dynamics of heavy-ion collisions, such high fluid flow velocities only occur for the small temperature region. Hence, setting $T_{0}$ to the maximum temperature encountered in the problem was found to give acceptable results.

Denoting the scaled momentum as $\bar{p}=p^{0} / T_{0}$, it proves convenient to further expand the coefficients $a^{(n)}$ in terms of generalized Laguerre polynomials $L_{k}^{(\alpha)}$, so that the complete ansatz for $f$ is given by

$$
f(t, x, p)=e^{-\bar{p}} \sum_{k=0}^{N_{p}-1} \sum_{n=0}^{N_{v}-1} P_{i_{1} \ldots i_{n}}^{(n)}(\mathbf{v}) L_{k}^{(\alpha)}(\bar{p}) a_{i_{1} \ldots i_{n}}^{(n k)}(t, x),
$$

where the choices $\alpha=2,3$ will be most relevant, so that $\alpha$ is restricted to integer numbers hereafter. For convenience, the main properties of Laguerre polynomials are reported in appendix B Using orthogonality, the coefficients $a^{(n k)}$ up to second order read as follows:

$$
\begin{aligned}
& \frac{m !}{(m+\alpha) !} \int d \bar{p} \bar{p}^{\alpha} \int \frac{d \Omega}{4 \pi} f P^{(0)} L_{m}^{(\alpha)}(\bar{p})=a^{(0 m)}(t, x), \\
& \frac{3 m !}{(m+\alpha) !} \int d \bar{p} \bar{p}^{\alpha} \int \frac{d \Omega}{4 \pi} f P_{i}^{(1)} L_{m}^{(\alpha)}(\bar{p})=a_{i}^{(1 m)}(t, x), \\
& \frac{15 m !}{2(m+\alpha) !} \int d \bar{p} \bar{p}^{\alpha} \int \frac{d \Omega}{4 \pi} f P_{i j}^{(2)} L_{m}^{(\alpha)}(\bar{p})=a_{i j}^{(2 m)}(t, x)(14)
\end{aligned}
$$

where we have used the fact that $a_{i j}^{(2 m)}$ may be taken to be traceless and symmetric.

The ideal choice for the discretization of velocity space is once again given by the requirement that the velocity moments (5) be represented exactly. Use of non Cartesian coordinates, however, implies that the associated lattice structure is no longer space-filling in general.

For a polynomial $P(\bar{p})$ of degree less than $2 N_{p}$, the integral over $\bar{p}$ can be recast into an exact sum

$$
\int d \bar{p} \bar{p} e^{-\bar{p}} P(\bar{p})=\sum_{i=0}^{N_{p}-1} \omega_{i}^{p} P\left(\bar{p}_{i}\right),
$$

if the nodes $\bar{p}_{0}, \ldots \bar{p}_{N_{p}-1}$ are given by $L_{N_{p}}^{(\alpha)}\left(\bar{p}_{i}\right)=0$ and the weights $\omega_{i}$ are given by

$$
\omega_{i}^{p}=\frac{\left(N_{p}+\alpha\right) !}{N_{p} !} \frac{\bar{p}_{i}}{\left(N_{p}+1\right)^{2}\left[L_{N_{p}+1}^{(\alpha)}\left(\bar{p}_{i}\right)\right]^{2}} .
$$

Note that the requirement (5) implies a polynomial $P(\bar{p})$ of degree $3-\alpha+N_{p}$, implying that $N_{p}>3-\alpha$ is a necessary condition to represent this polynomial exactly. The choice of $\alpha$ may depend on the problem, but $\alpha=3$ is particularly convenient here because it minimizes $N_{p}$. Unless one is interested in considering finite chemical potential, it is therefore convenient to choose $\alpha=3$, i.e. $N_{p}=1$, as we shall do from now on.

Coming to the angular dependence, for polynomials $P$ of $\sin \phi$ and $\cos \phi$ of degree less than $2 N_{\phi}$, integration over the polar angle $\phi$ can be expressed as

$$
\int_{-\pi}^{\pi} d \phi P(\phi)=\sum_{l=0}^{2 N_{\phi}-1} \omega_{l}^{\phi} P\left(\phi_{l}\right)
$$

where $\phi_{l}=\frac{l \pi}{N_{\phi}}$ and $\omega_{l}^{\phi}=\frac{\pi}{N_{\phi}}$. A polynomial $P^{(n)}\left(v^{i}\right)$ will only involve powers of $\cos \phi, \sin \phi$ up to $n$, so an exact representation of Eq. (5) requires $N_{v} \geq 3$, hence $N_{\phi} \geq 3$.

Finally, for the integration w.r.t to the cosine of the polar angle $\cos \left(\theta_{p}\right)=\xi$, there is a special symmetry that one can exploit. Namely, should the integrand contain a $p^{x} / p^{0}=\cos \phi \sqrt{1-\xi^{2}}$, then the $\phi$-integration will only give a non-zero value if another $\cos \phi$ is present in the integrand. The only way this can happen is through another factor $p^{x} / p^{0}$, which means that $\cos ^{2} \phi\left(1-\xi^{2}\right)$ must be present in the integrand. A similar argument may be given for $p^{y}$. Hence, the integral over the polar angle is always of the form $\int_{-1}^{+1} d \xi P(\xi)$, with $P(\xi)$ a polynomial of degree $2+N_{v}$ or less. This is represented accurately as

$$
\int_{-1}^{1} d \xi P(\xi)=\sum_{j=0}^{N_{\xi}-1} w_{j}^{\xi} P\left(\xi_{j}\right)
$$

for polynomial degrees less than $2 N_{\xi}$, $\xi_{j}$ being the roots of the Legendre Polynomial $P_{N_{\xi}}(x)$ and

$$
w_{j}^{\xi}=\frac{2}{\left(1-\xi_{j}^{2}\right)\left(P_{N_{\xi}}^{\prime}\left(\xi_{j}\right)\right)^{2}} .
$$

the corresponding weights.

The requirement (5) again implies $N_{\xi} \geq 3$.

To summarize, the requirements (5) are fulfilled for the ansatz (13) if one uses a discretized set of momenta

$$
p_{i j l}{ }^{\mu}=T_{0} \bar{p}_{i}\left(\begin{array}{c}
1 \\
\cos \phi_{l} \sqrt{1-\xi_{j}^{2}} \\
\sin \phi_{l} \sqrt{1-\xi_{j}^{2}} \\
\xi_{j}
\end{array}\right),
$$

with $\bar{p}_{i}$ the roots of $L_{N_{p}+1}^{(1)}, x_{j}$ the roots of $P_{N_{\xi}}$ and $\phi_{l}=$ $\frac{l \pi}{N_{\phi}}$, with $N_{p}, N_{\xi}, N_{\phi}$ greater or equal to 3 and $3 \leq N_{v} \leq$ $\min \left(N_{\phi}, N_{\xi}\right)$ (see Fig. 2 for illustration). 


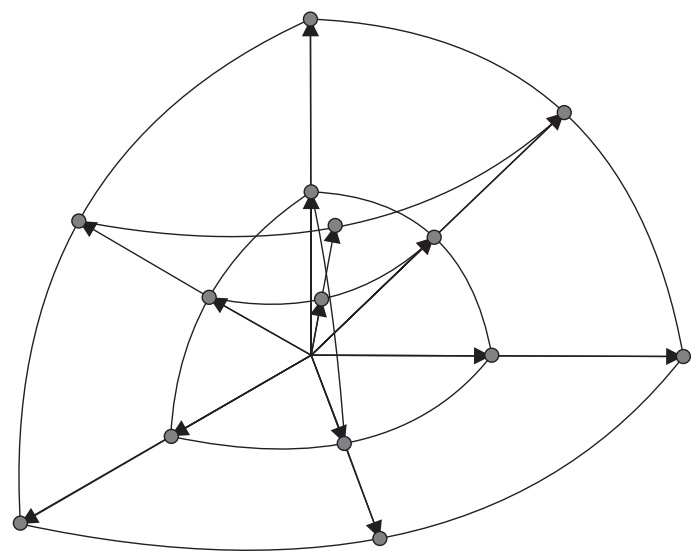

FIG. 2. Example of a lattice configuration for the present relativistic model. The arrows denote the discrete spatial components of $p^{\mu}$.

This implies a minimum number of 27 discrete "speeds" $p^{\mu}{ }_{i j l}$, quite comparable with the number of discrete speeds commonly used in non-relativistic LB theories.

With the momentum space thus discretized, we can move ahead and set up a concrete, fully relativistic lattice Boltzmann algorithm.

Before doing so, however, it is instructive to study how to extract the fluid velocity and local temperature distribution from a given distribution function $f \equiv f_{i j l}(t, x)$ discretized via (13).

\section{A. Energy-Momentum Conservation}

Using Eq. (13), the energy momentum tensor becomes

$$
T^{\mu \nu}=\frac{3 T_{0}^{4}}{\pi^{2}}\left(\begin{array}{cc}
a^{(00)} & a_{i}^{(10)} \\
a_{j}^{(10)} & a_{i j}^{(20)}+\frac{1}{3} \delta^{i j} a^{(00)}
\end{array}\right),
$$

The requirement (5) implies that $u^{\mu}$ should be a futurepointing eigenvector of the energy momentum tensor, or $u^{\mu} T_{\mu}^{\nu}=\epsilon u^{\nu}$. Thus, one has to calculate the eigensystem of $T_{\mu}^{\nu}$ and identify $u^{\mu}$ as the (only) future-pointing eigenvector, with eigenvalue $\epsilon$. Using existing numerical packages, this can be done in a rather efficient way. Once $\epsilon$ is known, the temperature is calculated from $\epsilon=\frac{3 T^{4}}{\pi^{2}}$. Since we neglected particle masses, the equation of state is always that of an ideal gas, or $P=\frac{\epsilon}{3}$. Non-ideal equations of state will be considered in a follow-up work.

\section{B. The equilibrium distribution function}

While the full equilibrium distribution function is given by Eq.(2), the LB algorithm approximates all $f$ 's by the ansatz (13). Hence, for consistency, $f^{\text {eq }}$ is also expanded as follows:

$$
\begin{aligned}
f^{\mathrm{eq}}(t, x, p)= & e^{-\bar{p}}\left(a_{\mathrm{eq}}^{(00)}(t, x)+P_{i} a_{i, \mathrm{eq}}^{(10)}(t, x)+P_{i j} a_{i j, \mathrm{eq}}^{(20)}(t, x)\right. \\
& \left.+P_{i j k} a_{i j k, \mathrm{eq}}^{(30)}(t, x)\right),
\end{aligned}
$$

where we neglect higher order terms. The coefficients are readily evaluated to be

$$
\begin{aligned}
a_{\mathrm{eq}}^{(00)} & =\left(1+\frac{4}{3} \mathbf{u}^{2}\right) \theta^{-4}, \\
a_{i, \mathrm{eq}}^{(10)} & =4 u^{i} u^{0} \theta^{-4}, \\
a_{i j, \mathrm{eq}}^{(20)} & =10\left(u^{i} u^{j}-\mathbf{u}^{2} \frac{\delta_{i j}}{3}\right) \theta^{-4}, \\
a_{i j k, \mathrm{eq}}^{(30)} & =\frac{35}{12 \mathbf{u}^{6}} P_{i j k}^{(3)}(u)\left(u^{0}\left(15-10 \mathbf{u}^{2}+8 \mathbf{u}^{4}\right)\right. \\
& \left.-\frac{15}{|\mathbf{u}|} \log \left(1+2 \mathbf{u}^{2}+2|\mathbf{u}| u^{0}\right)\right) \theta^{-4} .
\end{aligned}
$$

With these expressions at hand, we are now ready to construct an operational algorithm.

\section{THE FULLY RELATIVISTIC LB ALGORITHM}

First of all, a lattice version of the Boltzmann equation (1) needs to be established. For this purpose, we define three steps: i) Streaming in configuration space $(x$-move), ii) Streaming in momentum space ( $p$-move), iii) Collisional relaxation. These read as follows (discrete indices are relaxed for notational simplicity):

$$
\begin{aligned}
f^{\prime}\left(t, x^{i}, p^{\alpha}\right) & =f\left(t+\delta t, x^{i}+\frac{p^{i}}{p^{0}} \delta t, p^{\alpha}\right) \\
f^{\prime \prime}\left(t, x^{i}, p^{\alpha}\right) & =f\left(t, x^{i}, p^{\alpha}\right)+\delta t \Gamma_{\mu \nu}^{\lambda} \frac{p^{\mu} p^{\nu}}{p^{0}} \partial_{\lambda}^{(p)} f\left(t, x^{i}, p^{\alpha}\right), \\
f^{\prime}\left(t, x^{i}, p^{\alpha}\right) & =f^{\prime \prime}\left(t, x^{i}, p^{\alpha}\right)-\frac{p^{\mu} u_{\mu} \delta t}{p^{0} \tau_{R}}\left(f^{\prime \prime}-f^{\mathrm{eq}}\right) .
\end{aligned}
$$

Here, we use the notation $f\left(t, x^{i}, p^{\alpha}\right) \equiv f(t, x, p)$ to make the vector components explicit.

The first step consists in the streaming of the distribution functions according to the discrete momenta. The second one, is the implementation of the external forces due to the curvature of the space-time, and the third one is the collision step, expressed in terms of relaxation towards the local equilibrium.

The relativistic lattice Boltzmann algorithm is given by the following sequence of steps:

\section{Initialization:}

At the initial step, $f^{\prime}$ needs to be known at grid sites $x^{i}$ and discretized momenta $p^{\alpha}$. For this, we introduce the initial conditions of the specific problem and the initial distributions is typically specified as the local equilibria associated with the initial 
hydrodynamic fields $f\left(t=t_{0}, x^{i}, p^{\alpha}\right)=f^{e q}(t, x, p)$, where the equilibrium distribution is given by the expression (18).

2. $x$-move:

Calculate the new $f$ from

$$
f\left(t, x^{i}, p^{\alpha}\right)=f^{\prime}\left(t-\delta t, x^{i}-v^{i} \delta t, p^{\alpha}\right) .
$$

In non-relativistic lattice Boltzmann methods, this step is known as streaming, each populations moves to the site pointed by its corresponding discrete speed.

3. p-move:

Compute the change in the distribution function because of external/internal forces. In principle, a force term implies a change in velocity, thereby jeopardizing the discrete structure of velocity space. However, this can be preserved by moving the information according to the streaming step given above (with constant speeds/momenta) and then correcting the distribution form with an appropriate source term. The latter is identified by representing the derivative in momentum space also as a polynomial expansion:

$S(t, x) \equiv F^{\lambda} \partial_{\lambda}^{(p)} f=e^{-\bar{p}} \sum_{n=0}^{N_{v}-1} P_{i_{1} \ldots i_{n}}^{(n)}(\mathbf{v}) s_{i_{1} \ldots i_{n}}^{(n)}(t, x)$,

with $F^{\lambda}=\Gamma_{\mu \nu}^{\lambda} \frac{p^{\mu} p^{\nu}}{p^{0}}$.

The unknown source coefficients $s^{(n)}$ can be computed by inverting (20) and using integration by parts. Note that in general these coefficients can be expressed as sums over the coefficients $a^{(n 0)}$ in Eq. (13).

With the source term available, we evaluate $f^{\prime \prime}$ as

$$
\begin{aligned}
f^{\prime \prime}\left(t, x^{i}, p^{\alpha}\right) & =f\left(t, x^{i}, p^{\alpha}\right) \\
& +\delta t e^{-\bar{p}} \sum_{n=0}^{N_{v}-1} P_{i_{1} \ldots i_{n}}^{(n)}(\mathbf{v}) s_{i_{1} \ldots i_{n}}^{(n)}(t, x) .
\end{aligned}
$$

This step accounts for the geometric forces in the given space-time.

\section{Equilibration:}

In order to perform collisional relaxation, local discrete equilibria must be constructed first. To this purpose, we calculate the energy momentum tensor $T^{\mu \nu}$ corresponding to $f^{\prime \prime}$ from Eqs. (17) and (13). We then compute the values of the fluid 4-velocity $u^{\mu}$ and energy density $\epsilon$ by calculating the futurepointing eigenvector of $T^{\mu \nu}$. The local temperature is obtained by the equation of state. Using $T, u^{\mu}$, we calculate $f^{\text {eq }}$ from Eq. (18), and the collision coefficient $\Omega=p^{\mu} u_{\mu} /\left(p^{0} \tau_{R}\right)$.

\section{Collision:}

Calculate the post-collisional state $f^{\prime}$ from the known values of $f^{\prime \prime}, f^{\text {eq }}$, and $\Omega$, according to

$$
\begin{aligned}
f^{\prime}\left(t, x^{i}, p^{\alpha}\right) & =f^{\prime \prime}\left(t, x^{i}, p^{\alpha}\right)(1-\Omega \delta t) \\
& +\Omega \delta t f^{\mathrm{eq}}\left(t, x^{i}, p^{\alpha}\right) .
\end{aligned}
$$

6. Cycle through $2-5$ for each time-step until completion of the time evolution.

A remark concerning the $x$-move step is in order. Since the unit vectors $v^{i}=p^{i} /|\mathbf{p}|$ discretize the unit sphere (see Fig. 2), the displaced positions $x^{i}-v^{i} \delta t$ will typically not correspond to a neighbouring grid site, unless very particular geometries (e.g. a hexagonal lattice) are chosen. This breaks the light-cone rule discussed previously.

At least two ways out of this problem can be envisaged.

The first is to acknowledge the fact that spatial and momentum discretization can no longer be kept in synchrony, going back to the original Boltzmann equation (1) and discretize space derivatives in a flux-conserving way, according to one's favored finite-volume/difference practice. This is similar to the non-relativistic LB method on so-called unstructured meshes, wherein powerful features of modern finite-volume techniques are imported within the LB framework. For more details about this technique, we refer the reader to the literature, e.g. Refs. [43, 44]. The second method, the one we will adopt in the following, consists of transferring off-grid distributions into grid locations through (bilinear) interpolation.

Either way, it is clear that none of the two methods above can match the simplicity, hence computational efficiency, of the space-filling Cartesian formulation. In particular, they cannot preserve the exact nature of the streaming step in light-cone form. This limitation appears to be inherent to the non-separability of the relativistic Jüttner equilibria along the three spatial coordinates. In this respect, a complete transfer of the key assets of the non-relativistic scheme, does not appear to be feasible. This is the price to pay for a fully relativistic lattice formulation. However, since information is still traveling along constant streamlines in configuration space, this does not prevent the fully relativistic LB algorithm from delivering competitive performance, as we shall demonstrate in the next section, where concrete test-case simulation are presented.

\section{APPLICATION: THE BOOST-INVARIANT QUARK-GLUON PLASMA}

As a first application, let us consider the Milne spacetime generated by the coordinate transformation $\tau=$ $\sqrt{t^{2}-z^{2}}, Y=\tanh ^{-1}(z / t)$. In these coordinates, the metric takes the form

$$
g_{\mu \nu}=\operatorname{diag}\left(g_{\tau \tau}, g_{x x}, g_{y y}, g_{Y Y}\right)=\operatorname{diag}\left(1,-1,-1,-\tau^{2}\right)
$$


where hereafter the $(+,-,-,-)$ sign convention is assumed. In this metric, the non-vanishing Christoffel symbols are:

$$
\Gamma_{\tau T}^{Y}=\Gamma_{Y \tau}^{Y}=\frac{1}{\tau}, \quad \Gamma_{Y Y}^{\tau}=\tau .
$$

This implies a non-vanishing covariant fluid divergence even for a fluid a rest, $u^{\mu}=(1, \overrightarrow{0})$, i.e.

$$
\nabla_{\mu} u^{\mu}=\partial_{\mu} u^{\mu}+\Gamma_{\mu \nu}^{\mu} u^{\nu}=\Gamma_{Y \tau}^{Y} u^{\tau}=\frac{1}{\tau} \neq 0 .
$$

The reason for this is that the Milne space-time is expanding in one dimension, so that a system at rest experiences the 'stretching' of space-time. This is a nice feature, because it naturally mimics the expansion of the system, following a heavy-ion collision in Minkowski space (see Ref. 24], Section 5B for details). In Minkowski space-time, a general solution to $p^{\mu} \partial_{\mu} f=0$ is e.g. $f=f\left(p_{\perp}, t \vec{p}-\vec{x} p^{t}\right)$. In Milne-coordinates that would correspond to

$$
f=f\left(p_{\perp}, \tau^{2} p^{Y}, \cosh Y\left(\tau \vec{p}_{\perp}-\vec{x}_{\perp} p^{\tau}\right)-\tau p^{Y} \vec{x}_{\perp} \sinh Y\right) .
$$

This can be further simplified by considering only the evolution at mid-rapidity, $Y \simeq 0$. It is readily checked that, under such condition, the action of the derivative $\partial_{\tau}^{(p)}$ is exactly cancelled by the $\partial_{Y}$ derivative of the last term in (22). One may thus neglect both, so that the Boltzmann equation at mid-rapidity simplifies to

$$
\left[p^{\tau} \partial_{\tau}+\vec{p}_{\perp} \cdot \vec{\partial}_{\perp}\right] f-2 \frac{\partial f}{\partial p^{Y}} \Gamma_{Y \tau}^{Y} p^{Y} p^{\tau}=-\mathcal{C}[f], \quad Y \simeq 0
$$

where $p^{\tau}$ is treated as an independent variable (e.g. $\left.\partial_{Y}^{(p)} p^{\tau}=0\right)$. The corresponding discrete version of the lattice Boltzmann equation takes the following form:

$$
\begin{aligned}
f\left(\tau+\delta \tau, x^{i}+\frac{p^{i}}{p^{\tau}} \delta \tau, p^{\alpha}\right) & =f^{\prime}\left(\tau, x^{i}, p^{\alpha}\right), \\
f^{\prime \prime}\left(\tau, x^{i}, p^{\alpha}\right) & =f\left(\tau, x^{i}, p^{\alpha}\right)+\frac{2 \delta \tau}{\tau} p^{Y} \partial_{Y}^{(p)} f, \\
f^{\prime}\left(\tau, x^{i}, p^{\alpha}\right) & =f^{\prime \prime}\left(\tau, x^{i}, p^{\alpha}\right) \\
& -\frac{p^{\mu} u_{\mu} \delta \tau}{p^{\tau} \tau_{R}}\left(f^{\prime \prime}-f^{\mathrm{eq}}\right) .
\end{aligned}
$$

\section{A. Warmup: 0+1dimensions in Milne space-time}

The simplest practical example is given by considering a system that is homogeneous in the Milne coordinates, e.g. $f=f\left(\tau, p^{\alpha}\right)=f\left(\tau, \bar{p}, \xi_{i}\right)$. Because there is no spacedependence left, one may use a simplified version of the discretization ansatz (13),

$$
f\left(\tau, p^{\alpha}\right)=e^{-4} \sum_{k=0}^{N_{\xi}-1} a_{0 k}(\tau) P_{k}(\xi),
$$

where $p^{z}=p^{Y} \tau$, such that $p^{\tau}=\sqrt{p_{\perp}^{2}+\left(p^{Y} \tau\right)^{2}}$ becomes $p^{\tau}=|\mathbf{p}|=\sqrt{p_{\perp}^{2}+p_{z}^{2}}$ and $\xi=\arccos \frac{p^{z}}{p^{\tau}}$. With $f$ discretized this way, one readily inverts to obtain the coefficients $a_{m l}$. For instance

$$
\begin{aligned}
a_{0 l} & =\frac{2 l+1}{12} \int_{0}^{\infty} d \bar{p} \bar{p}^{3} \int d \xi P_{l}(\xi) f(\tau, \bar{p}, \xi) \\
& =\frac{2 l+1}{2} e^{4} \sum_{j=0}^{N_{\xi}-1} \omega_{j}^{\xi} P_{l}\left(\xi_{j}\right) f\left(\bar{p}_{i}, \xi_{j}\right),
\end{aligned}
$$

where the second line is the discretized version and we recall that $\xi_{j}$ are the roots of $P_{N_{\xi}}$ and the weights $\omega^{\xi}$ were specified above. Starting with an initial equilibrium distribution function

$$
f\left(\tau_{0}, \bar{p}, \xi_{i}\right) \propto e^{-\bar{p} / \theta},
$$

and discretizing $f\left(\tau_{0}\right)$ on a $\xi$ grid with $N_{\xi}$ points $(\bar{p}=4)$, the algorithm reads as follows.

First set $f^{\prime}=f$. Then, set

$$
p^{z} \partial_{z}^{(p)} f=e^{-\bar{p}}\left(S^{0}+S^{2} P_{2}(\xi)\right),
$$

where the coefficients are calculated to be

$$
\begin{aligned}
S^{0} & =-\frac{1}{12} \int d \bar{p} \bar{p}^{3} \int d \xi\left(1+\xi^{2}\right) f, \\
S^{2} & =-\frac{5}{24} \int d \bar{p} \bar{p}^{3} \int d \xi\left(3 \xi^{4}-8 \xi^{2}+1\right) f .
\end{aligned}
$$

Calculate $f^{\prime \prime}$ from

$f^{\prime \prime}\left(\tau_{0}+\delta \tau, \bar{p}, \xi_{i}\right)=f\left(\tau_{0}, \bar{p}, \xi_{i}\right)+\frac{\delta \tau}{\tau+\delta \tau} e^{-\bar{p}}\left(S^{0}+S^{2} P_{2}(\xi)\right)$.

Next, calculate the equilibrium temperature via the energy-momentum tensor corresponding to $f^{\prime \prime}$ and obtain $f^{\prime}\left(\tau_{0}+\delta \tau\right)$ from Eq.(21).

The above steps are cycled in time over the prescribed time-span of the simulation.

\section{B. Comparison with exact results}

Let us now compare the results of the above lattice Boltzmann algorithm in Milne-spacetime against known exact results. First, let us consider the case in which the relaxation time is so large that the collision term plays a negligible role. The solution to the Boltzmann equation must therefore be very close to the free streaming solution,

$$
f_{\text {free-stream }}(\tau, p, \xi)=e^{-p \sqrt{1+\xi^{2} r} / Q},
$$

where $r=\frac{\tau^{2}}{\tau_{0}^{2}}-1$ and $Q$ is the initial temperature scale. From this, the temperature can be obtained from the energy-momentum tensor as

$$
T(\tau)=Q\left[\frac{1}{2}\left(\frac{\tau_{0}^{2}}{\tau^{2}}+\frac{\arctan \sqrt{\tau^{2} / \tau_{0}^{2}-1}}{\sqrt{\tau^{2} / \tau_{0}^{2}-1}}\right)\right]^{1 / 4},
$$


As opposed to the free-streaming case, let us now consider the opposite extreme of small viscosity, i.e. very fast relaxation to the local equilibrium. In this case, the 'exact' temperature evolution is given by fluid dynamics. More specifically, denoting $T_{Y}^{Y}-P \equiv \Phi$, the energy density and $\Phi$ fulfill the coupled equations [24]

$$
\begin{aligned}
\partial_{\tau} \epsilon & =-\frac{\epsilon+P}{\tau}+\frac{\Phi}{\tau} \\
\partial_{\tau} \Phi & =-\frac{\Phi}{\tau_{\pi}}+\frac{4 \eta}{3 \tau_{\pi} \tau}-\frac{4 \Phi}{3 \tau}-\frac{\lambda_{1}}{2 \tau_{\pi} \eta^{2}} \Phi^{2}
\end{aligned}
$$

where $\tau_{\pi}$ is the relaxation time and $\lambda_{1}$ is a self-coupling parameter that can be calculated [25], presumably fairly easily for the BGK collision kernel used here.

Since both are second-order corrections to hydrodynamics, their determination is left for future work. Here, we simply set $\lambda_{1}=\frac{6 \eta^{2}}{(\epsilon+P)}$ and vary $\tau_{\pi}$ between $\tau_{\pi}=$ $\frac{2(2-\ln 2)}{T} \frac{\eta}{s}$ and $\tau_{\pi}=\frac{6}{T} \frac{\eta}{s}$ (the weak and strong coupling limit, respectively, [21]). The above set of hydrodynamic equations display simple analytic solutions for the case of vanishing viscosity (ideal fluid) and first order gradient expansion (Navier-Stokes). These are [20, 26] given by

$$
T(\tau)=T_{0}\left(\frac{\tau_{0}}{\tau}\right)^{1 / 3}\left[1+\frac{2 \eta}{3 s \tau_{0} T_{0}}\left(1-\left(\frac{\tau_{0}}{\tau}\right)^{2 / 3}\right)\right] .
$$

The full set of equations (27) is second-order in gradients and thus causal for $\tau_{\pi}$ larger than some critical value. For general values of $\tau_{\pi}, \lambda_{1}$, it has to be solved numerically.

In Fig 3, we show a comparison between the lattice Boltzmann algorithm, against various 'exact' results for the case of $\eta / s=0.5$ and $T_{0}\left(\tau_{0}=1 \mathrm{fm} / \mathrm{c}\right)=0.5 \mathrm{GeV}$.

The second-order set of hydrodynamic equations was solved using forward time differencing $\delta \tau \partial_{\tau} X(\tau)=$ $(X(\tau+\delta \tau)-X(\tau))$. A time step of $\delta \tau=0.01 \tau_{0}$ was required to reach a stable continuum result. Conversely, for the lattice Boltzmann algorithm, typically the result is stable for $\delta \tau<0.2 \tau_{0}$, nearly 20 times larger than the fluid dynamics requirement and only about 5 times smaller than standard LB schems in cartesian geometries. Based on the general arguments discussed earlier on in this paper, we interpret this encouraging outcome as the beneficial effect of moving information along constant streamlines.

As can be seen from this figure, the lattice Boltzmann algorithm does track the $2^{\text {nd }}$ order viscous fluid dynamics result from early times onwards. (The Navier-Stokes result has a different initial condition and hence the other results are not expected to track this curve). The numbers in Fig. 3 were chosen such that the initial temperature corresponds to the maximum temperature expected for heavy-ion collisions at $\sqrt{s}=5.5 \mathrm{TeV}$ at the Large Hadron Collider. Moreover, $T=0.15 \mathrm{GeV}$ is the temperature where a freeze-out to hadrons is expected. Hence, for this temperature region, the lattice Boltzmann algorithm with $N_{\xi}=5$ accurately reproduces the $2^{\text {nd }}$ order viscous fluid dynamics results.
Temperature

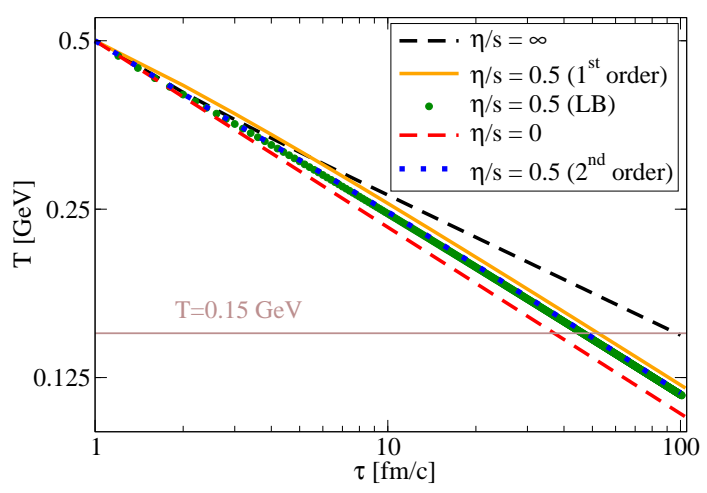

Scaled Temperature

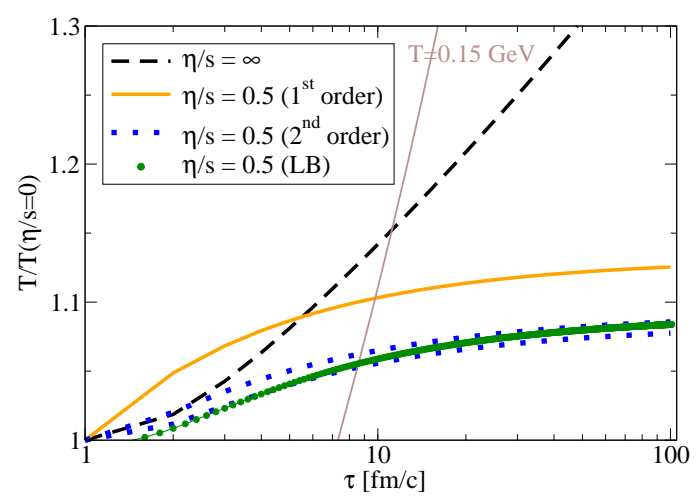

FIG. 3. Temperature evolution of a system starting at $\tau_{0}=1$ $\mathrm{fm} / \mathrm{c}$ and temperature $T_{0}=0.5 \mathrm{GeV}$. Results shown are $1^{\text {st }}$ order viscous fluid dynamics (Navier-Stokes) (initially not in equilibrium), $2^{\text {nd }}$ order viscous fluid dynamics (two values of $\tau_{\pi}$, see text), and the lattice Boltzmann result (LB) for $N_{\xi}=$ 5 , all for $\eta / s=0.5$. As a reference, the free streaming result $(\eta / s \rightarrow \infty)$ and the result for ideal fluid dynamics $(\eta / s=0)$ are also shown (top panel). Bottom panel: results are divided by the ideal fluid dynamic result to highlight differences. The $\mathrm{LB}$ result is seen to converge to the $2^{\text {nd }}$ order viscous fluid dynamics.

The dependence of the lattice Boltzmann result on the chosen discretization is shown in Fig, There, one can see that all cases (even $N_{\xi}=3$ ) reproduce the viscous fluid result, and for finer discretization $N_{\xi} \geq 4$, the LB results are indistinguishable to the naked eye. The viscosity dependence is also shown in Fig 4 The lattice Boltzmann algorithm tracks the fluid dynamic result for viscosities up to $\eta / s \sim 1.0$. For smaller viscosities, $\eta / s<0.5$, LB undershoots the fluid dynamics result. However, decreasing $\delta \tau$ by a factor two, brings the LB back in line with the fluid dynamic result. 


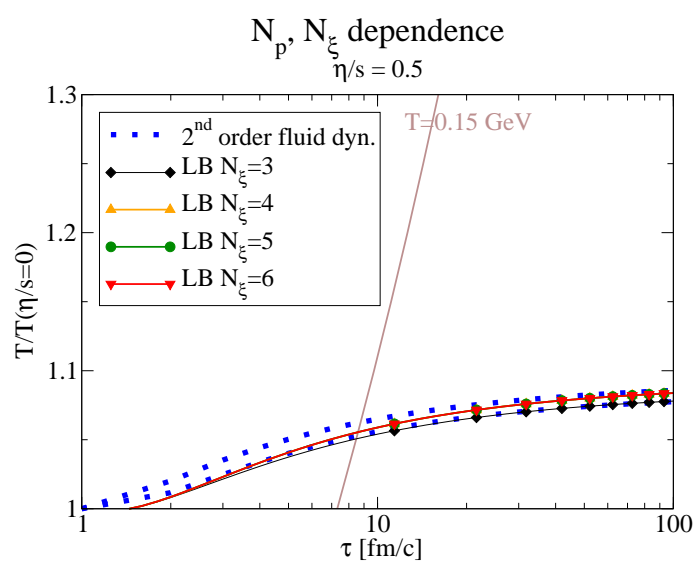

$\eta / \mathrm{s}$ dependence

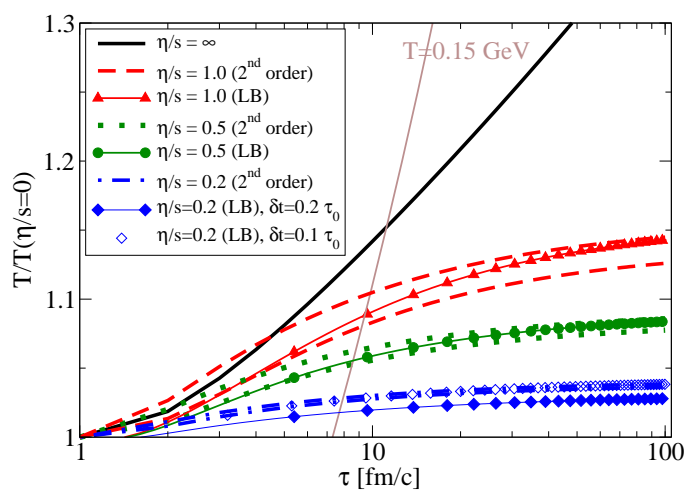

FIG. 4. Top: dependence of temperature evolution on discretization (for $\eta / s=0.5$ ). Bottom: dependence of result on value of shear viscosity coefficient compared to $2^{\text {nd }}$ order viscous fluid dynamics (for $N_{\xi}=5$ ). Viscous fluid results from (27) for two values of $\tau_{\pi}$, see text. For the lowest viscosity shown $(\eta / s=0.2)$ we highlight the effect of numerical viscosity by choosing two different values of $\delta \tau$.

\section{2+1 dimension in Milne space-time}

Next we consider the case in which the transverse dynamics is also taken account. Inclusion of the transverse space dynamics requires the solution of Eq. (24), using the full discretization (13). For convenience, we choose a square lattice for the grid in $x_{\perp}$. Choosing furthermore $\delta \tau=\delta x$, we use bilinear interpolation to obtain $f^{\prime}$ at points that lie in-between lattice sites. The change in momenta $p$ is calculated similar to Eq.(20).

The LB solver is applied to simulate the evolution of the medium created in $A u+A u$ collisions at top RHIC energies $(\sqrt{s}=200 \mathrm{GeV})$. For smooth initial conditions, the results may then be compared to the fluid dynamics solution, given by the code $\mathrm{VH} 2+1[6,27]$. This code has been cross-tested against several other codes and is generally credited for producing reliable results for smooth initial conditions.

The initial conditions are generated at initial time $\tau=$
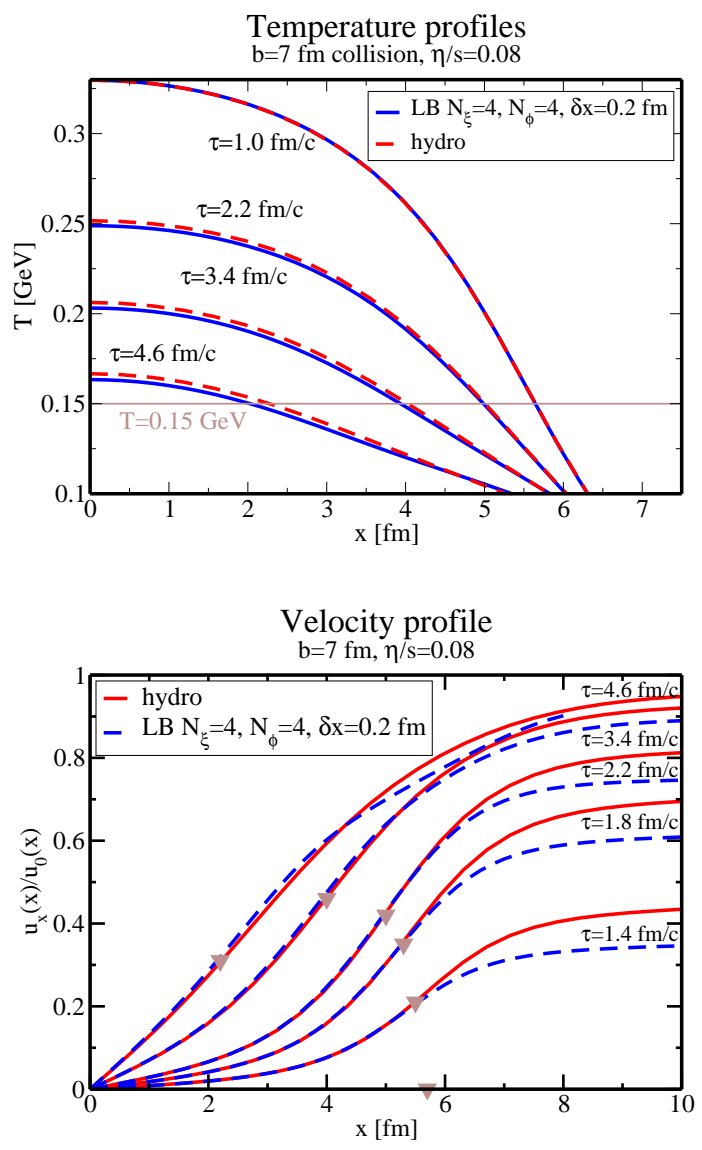

FIG. 5. Top: temperature evolution in viscous hydrodynamics ('hydro') versus lattice Boltzmann equation ('LB') for $\eta / s=0.08$. As can be seen, the temperature evolution in the lattice Boltzmann approach for $\delta x=0.2 \mathrm{fm}$ is reasonably close to the 'exact' hydrodynamic result. Bottom: evolution of velocity $u^{x} / u^{0}$ in viscous hydrodynamics versus LB. Even high velocities up to 80 percent of the speed of light are well represented, but at later times an instability develops in the low temperature region (a grey triangle marks the position of $T=0.15 \mathrm{GeV}$ ). Note that the discrepancy at larger $x$ lies exclusively in the region $T<0.15 \mathrm{GeV}$ (compare top plot).

$1 \mathrm{fm} / \mathrm{c}$ from a Glauber model, with number of collisions scaling (c.f. 24]) on a $69 \times 69,139 \times 139$ or $279 \times 279$ grid with a lattice spacing of $\delta x=0.4 \mathrm{fm}, \delta x=0.2 \mathrm{fm}$ or $\delta x=0.1 \mathrm{fm}$, respectively. The maximum temperature at the center of the grid is $T^{\max }=0.37 \mathrm{GeV}$ for central collisions (impact parameter $b=0 \mathrm{fm}$ ).

In Fig. VIIC, the temperature evolution from LB with $N_{\xi}=4, N_{\phi}=4$ for $\delta x=0.2 \mathrm{fm}$ is compared to the $\mathrm{VH} 2+1$ solution. As can be seen from this figure, the LB code reproduces the $\mathrm{VH} 2+1$ result rather accurately for temperatures $T \geq 0.15 \mathrm{GeV}$, the relevant temperature regime for the fluid medium. At later times $\tau>5 \mathrm{fm} / \mathrm{c}$, when all fluid cells have cooled below a temperature of $0.15 \mathrm{GeV}$, numerical instabilities develop at the outer edges, for the discretization used $N_{\xi}=4, N_{\phi}=4$. We 
have checked that the remaining discrepancy between fluid dynamics and LB in the temperature evolution close to $x \sim 0$ can be cured by using a smaller lattice spacing $\delta x=0.1 \mathrm{fm}$.

Also shown in Fig. VIIC is the evolution of the velocity $\frac{u_{x}}{u_{0}}$. One can see that LB tracks the $\mathrm{VH} 2+1$ result closely for the high temperature region (there are clear deviations at low temperatures $T<0.15 \mathrm{GeV}$, compare left plot in Fig. VIIC). Even high velocities seem to be well represented, but the algorithm does not handle correctly velocities $u_{x} / u_{0}>0.8$. Presumably, this discrepancy can be cured by including higher order terms in Eq.(18). However, it should be pointed out that for the high temperature region $T>0.15$, the velocity distributions from fluid dynamics are accurately reproduced.

\section{CONCLUSIONS}

Summarizing, we have developed a new scheme based on the lattice-Boltzmann method to model relativistic fluid dynamics in general spacetime. The main advantage of our scheme, as compared with previous relativistic lattice Boltzmann models [39, 40], rests mainly with its ability describe the dynamics of ultra-relativistic systems in general space-time geometries. The present model differs from typical lattice Boltzmann schemes mostly in the streaming step, which, because of the spherical shape of the discrete momenta, is no longer space-filling. Instead, multi-linear interpolation is used to represent the distribution functions in the second-nearest neighbours of each cell on the lattice. This interpolation breaks the exact nature of the standard LB streaming operator. However, at variance with hydrodynamic formulations, it still moves information along constant streamlines, thereby permitting to march in larger time-steps than hydrodynamic codes. Our scheme has been validated through simulations in quark-gluon plasma, yielding very satisfactory agreement with other computational methods based on a macroscopic description, at a lower computational cost (nearly two orders of magnitude faster than the $\mathrm{VH} 2+1$ viscous hydro code 6] and still a factor $3-5$ as compared to optimized ones [45]).

Because of these favorable properties, we expect this new LB method to offer a new competitive entry for the computational study of large-scale complex relativistic fluids.

\section{ACKNOWLEDGEMENTS}

We would like to thank G. Denicol and H. Herrmann for interesting discussions. This work was supported in part by the Helmholtz International Center for FAIR within the framework of the LOEWE program launched by the state of Hesse.

\section{Appendix A: The $P^{(n)}$ Legendre polynomials}

The vector polynomials $P_{i_{1} \ldots i_{n}}^{(n)}(\mathbf{v})$ are constructed by requiring orthogonality with respect to the angular integral $\int \frac{d \Omega}{4 \pi}$. One finds Specifically, the polynomials involved are given by

$$
\begin{aligned}
P^{(0)} & =1, \\
P_{i}^{(1)} & =v_{i} \\
P_{i j}^{(2)} & =v_{i} v_{j}-\frac{1}{3} \delta_{i j}, \\
P_{i j k}^{(3)} & =v_{i} v_{j} v_{k}-\frac{1}{5}\left(\delta_{i j} v_{k}+\delta_{i k} v_{j}+\delta_{j k} v_{i}\right), \\
\ldots &
\end{aligned}
$$

The orthogonality relations for the first few polynomials are found to be

$$
\begin{aligned}
\int \frac{d \Omega}{4 \pi} P^{0} P^{0} & =1 \\
\int \frac{d \Omega}{4 \pi} P_{i}^{(1)} P_{j}^{(1)} & =\frac{\delta_{i j}}{3} \\
\int \frac{d \Omega}{4 \pi} P_{i j}^{(2)} P_{l m}^{(2)} & =\frac{1}{15}\left(\delta_{i l} \delta_{j m}+\delta_{i m} \delta_{j l}-\frac{2}{3} \delta_{i j} \delta_{l m}\right), \\
& \ldots
\end{aligned}
$$

\section{Appendix B: Generalized Laguerre Polynomials}

The first few generalized Laguerre Polynomials are given by

$$
\begin{aligned}
L_{0}^{(\alpha)}(x) & =1 \\
L_{1}^{(\alpha)}(x) & =1+\alpha-x, \\
L_{2}^{(\alpha)}(x) & =\frac{x^{2}}{2}-(\alpha+2) x+\frac{(\alpha+2)(\alpha+1)}{2}, \\
L_{3}^{(\alpha)}(x) & =-\frac{x^{3}}{6}+\frac{(\alpha+3) x^{2}}{2}-\frac{(\alpha+3)(\alpha+2) x}{2} \\
& +\frac{(\alpha+3)(\alpha+2)(\alpha+1)}{6} .
\end{aligned}
$$

The orthogonality relation is given by

$$
\int_{0}^{\infty} d x e^{-x} x L_{n}^{(\alpha)}(x) L_{m}^{(\alpha)}(x)=\frac{\Gamma(n+\alpha+1)}{n !} \delta_{n m} .
$$


[1] K. Adcox et al. [PHENIX Collaboration], Nucl. Phys. A 757, 184 (2005).

[2] B. B. Back et al., Nucl. Phys. A 757, 28 (2005).

[3] I. Arsene et al. [BRAHMS Collaboration], Nucl. Phys. A 757, 1 (2005).

[4] J. Adams et al. [STAR Collaboration], Nucl. Phys. A 757, 102 (2005).

[5] P. F. Kolb, U. W. Heinz, In *Hwa, R.C. (ed.) et al.: Quark gluon plasma* 634-714. nucl-th/0305084.

[6] P. Romatschke, U. Romatschke, Phys. Rev. Lett. 99 , 172301 (2007). arXiv:0706.1522 [nucl-th]].

[7] K. Dusling, D. Teaney, Phys. Rev. C77 , 034905 (2008). arXiv:0710.5932 [nucl-th]].

[8] H. Song, U. W. Heinz, Phys. Rev. C77 , 064901 (2008). arXiv:0712.3715 [nucl-th]].

[9] A. K. Chaudhuri, Phys. Lett. B672 , 126-131 (2009). arXiv:0803.0643 [nucl-th]].

[10] H. Niemi, G. S. Denicol, P. Huovinen et al., arXiv:1101.2442 [nucl-th]].

[11] J. L. Nagle, M. P. McCumber, arXiv:1011.1853 [nucl$\mathrm{ex}]$.

[12] D. Teaney, L. Yan, arXiv:1010.1876 [nucl-th]].

[13] M. Luzum, Phys. Lett. B696 , 499-504 (2011). arXiv:1011.5773 [nucl-th]].

[14] M. Luzum, J. -Y. Ollitrault, arXiv:1011.6361 [nucl-ex]].

[15] P. Sorensen, B. Bolliet, A. Mocsy et al., arXiv:1102.1403 [nucl-th]].

[16] B. Schenke, S. Jeon, C. Gale, Phys. Rev. Lett. 106 (2011) 042301. arXiv:1009.3244 [hep-ph]].

[17] M. Müller, J. Schmalian, L. Fritz, Phys. Rev. Let.103, 025301 (2009).

[18] P. Romatschke, Prog. Theor. Phys. Suppl. 174 , 137-144 (2008). arXiv:0710.0016 [nucl-th]].

[19] I. Fouxon, Y. Oz, Phys. Lett. B694 , 261-264 (2010). arXiv:0909.3574 [hep-th]].

[20] R. Baier, P. Romatschke, U. A. Wiedemann, Phys. Rev. C73 , 064903 (2006). hep-ph/0602249.

[21] R. Baier, P. Romatschke, D. T. Son et al., JHEP 0804 , 100 (2008). arXiv:0712.2451 [hep-th]].

[22] X. Shan, X. He, Phys. Rev. Lett. 80, 65 (1998).

[23] H. Grad, Commun. Pure Appl. Math. 2, 325 (1949).

[24] P. Romatschke, arXiv:0902.3663 [hep-ph].

[25] M. A. York, G. D. Moore, Phys. Rev. D79 , 054011 (2009). arXiv:0811.0729 [hep-ph]].
[26] A. Muronga, Phys. Rev. Lett. 88 , 062302 (2002). nucl-th/0104064.

[27] M. Luzum, P. Romatschke, Phys. Rev. C78 , 034915 (2008). arXiv:0804.4015 [nucl-th]].

[28] R. Benzi and S. Succi and M. Vergassola, Phys. Rep. 222, 145-197 (1992)

[29] He, Xiaoyi and Luo, Li-Shi, Phys. Rev. E 56, 6811-6817 (1997).

[30] Guy R. McNamara and Gianluigi Zanetti, Phys. Rev. Lett. 61, 23322335 (1988).

[31] Zhaoli Guo and T. S. Zhao, Phys. Rev. E 66, 036304 (2002).

[32] S. Succi and G. Amati and R. Piva, Int. J. Mod. Phys. C 8, 869 (1997).

[33] B. M. Boghosian and P.J. Love and P. V. Coveney and S. Succi and I. Karlin and J. Yepez, Phys. Rev. E Rapid Communications 68, 025103 Part 2 (2003).

[34] Shiyi Chen and Gary D. Doolen, Annual Review of Fluid Mechanics 30, 329-364 (1998).

[35] Anthony J. C. Ladd, Journal of Fluid Mechanics 271, 285-309 (1994).

[36] A. M. Artoli, D. Kandhai, H. C. J. Hoefsloot, A. G. Hoekstra and P. M. A. Sloot, Computational Science 2657, 665 (2003).

[37] Shiyi Chen, Hudong Chen, Daniel Martnez, and William Matthaeus, Phys. Rev. Lett. 67, 37763779 (1991).

[38] M. Mendoza and J. D. Muñoz, Phys. Rev. E 77, 026713 (2008).

[39] M. Mendoza, B. M. Boghosian, H. J. Herrmann, and S. Succi, Phys. Rev. Lett. 105, 014502 (2010).

[40] M. Mendoza, B. M. Boghosian, H. J. Herrmann, and S. Succi, Phys. Rev. Rev. D 82, 105008 (2010).

[41] C. Cercignani and G. M. Kremer, The Relativistic Boltzmann Equation: Theory and Applications (Boston; Basel; Berlin: Birkhauser, 2002).

[42] M. Mendoza, H. J. Herrmann, and S. Succi, Phys. Rev. Lett. 106, 156601 (2011).

[43] Gongwen Peng, Haowen Xi, and Comer Duncan, Phys. Rev. E 58, R4124R4127 (1998).

[44] S. Ubertini and S. Succi, Progress in Computational Fluid Dynamics, 5, 85, (2005).

[45] S. Pratt, D. Teaney, B. Schenke, private communications. 